\title{
Residential mobility impacts relative risk estimates of space-time clusters of chlamydia in Kalamazoo County, Michigan
}

\author{
Claudio Owusu, ${ }^{1}$ Michael R. Desjardins, ${ }^{1}$ Kathleen M. Baker, ${ }^{2}$ Eric Delmelle ${ }^{1}$ \\ ${ }^{1}$ Department of Geography and Earth Sciences and Center for Applied Geographic Information Science, \\ University of North Carolina at Charlotte, Charlotte; ${ }^{2}$ Department of Geography and Health Data Research, \\ Analysis and Mapping (HDReAM) Center, Western Michigan University, Kalamazoo, USA
}

\begin{abstract}
We determine the impact of residential mobility in the prevalence and transmission dynamics of sexually transmitted infections. We illustrate our approach on reported chlamydia infections obtained from the Michigan Disease Surveillance System for Kalamazoo County, USA, from 2006 to 2014. We develop two scenarios, one with fixed residential addresses and one considering residential mobility. We then compare the resulting space-time clusters and relative risk (RR) of infection. The space-time scan statistics showed increased RR in an area with previously low risk
\end{abstract}

Correspondence: Eric Delmelle, Department of Geography and Earth Sciences and Center for Applied Geographic Information Science, University of North Carolina at Charlotte, 9201 University City Boulevard, Charlotte, NC 28036, USA.

E-mail: Eric.Delmelle@uncc.edu

Key words: Sexually transmitted infections; Residential mobility; Chlamydia; Health; Space-time, USA.

Funding: none.

Note: this study was developed from thesis research when the first author was pursuing his master's degree. The first author thereafter sought consent from Health Data Research, Analysis and Mapping (HDReAM) Center at Western Michigan University to advance this work, leading to this paper. All protocols for this study was approved by WMU Human Subjects Institutional Review Board (14-09-27) as such, this publication will not in any way seek to identify the subjects in the data used in the analysis.

Received for publication: 6 September 2019.

Revision received: 1 October 2019.

Accepted for publication: 2 October 2019.

${ }^{\circ}$ Copyright: the Author(s), 2019

Licensee PAGEPress, Italy

Geospatial Health 2019; 14:812

doi:10.4081/gh.2019.812

This article is distributed under the terms of the Creative Commons Attribution Noncommercial License (CC BY-NC 4.0) which permits any noncommercial use, distribution, and reproduction in any medium, provided the original author(s) and source are credited. of sexually transmitted infections. In addition, even though the spatial extent of the three clusters identified did not change significantly at the scale we conducted our analysis at, the temporal extent (duration) did exhibit significant changes and could be considered for unique interventions. The results indicate that residential mobility has some dependency on the prevalence and transmission dynamics of sexually transmitted infections to new areas. We suggest that strategies adopted to reduce the burden of sexually transmitted infections take into consideration the relatively high residential mobility of at-risk populations to reduce spreading the infections to new areas.

\section{Introduction}

More than two million newly diagnosed cases of sexually transmitted infections (STIs - e.g. chlamydia, gonorrhoea and syphilis) were reported in the United States (U.S.) during 2016 (Centers for Disease Control and Prevention, CDC, 2017). Untreated STI can cause severe health effects such as pelvic inflammatory disease in women and apoptosis in men, resulting in an increase in infertility (Satta et al., 2005; Centers for Disease Control and Prevention, CDC, 2017). Beyond these health effects, the economic burden for the treatment of STIs in the U.S. is nearly $\$ 16$ billion per year (Owusu-Edusei Jr et al., 2013).

Over the past few decades, studies have found that high incidences of STIs are driven by a high prevalence of infection among core groups (Bernstein et al., 2004; Bush et al., 2008; Gesink et al., 2011; Owusu et al., 2018). Core groups often have repeated infections and are associated with commercial sex workers (Nguyen et al., 2009; Neaigus et al., 2016), homosexual men (van Aar et al., 2016) or simply residents of low socioeconomic areas (Thomas et al., 2010; Owusu et al., 2018). These individuals often have structured networks of sexual partnerships with high sexual behaviours (Zenilman et al., 1999; Gunn et al., 2000; Bernstein et al., 2004).

Individuals in the core group tend to recruit new sexual partners into their network periodically, particularly when they change their geographic residence (Aral, 2000; Stratford et al., 2000; Lichtenstein et al., 2008; Cassels et al., 2017). Few studies have identified this pattern in truck drivers, commercial sex workers and migrant labourers (Stratford et al., 2000; Lichtenstein et al., 2008; Nguyen et al., 2009; Neaigus et al., 2016; Cassels et al., 2017). These studies provide evidence suggesting that transmission of STI to new areas are related to the mobility of individuals in some occupations. However, most of the studies suffer from small sample sizes which undermine the generalisation of the role 
of mobility in the spread of STIs among a large population with unknown occupations. In that case, we can resort from understanding the transmission dynamics of STI in a large population by assessing the residential mobility of individuals reported in the surveillance data.

Residential mobility is the movement of individuals among residential addresses within a year or over a period of years (Stack, 1994; Jacquez et al., 2005, 2007, 2013). This is often characterised as the frequency of moves over time (Stack, 1994; Jacquez et al., 2005, 2007, 2013). High rates of residential mobility have been associated with poor health outcomes such as cancer (Jacquez et al., 2013), cannabis use (Fergusson and Horwood, 1997; Morris et al., 2016), increased behavioural risk in children (Jelleyman and Spencer, 2008), and teenage pregnancy (Stack, 1994). Incorporating residential mobility in health studies enable identification of changes in disease clusters and further understanding of disease transmission dynamics (Jacquez et al., 2007, 2013). This information is critical for cost-effective, targeted interventions for purposes of protecting public health.

When examining longitudinal data, space-time clustering techniques have also emerged as a useful approach to understand the long-term dynamics of infectious disease, which cannot be addressed using solely spatial clustering approaches. Because, space-time clustering approaches can estimate and examine the size, duration, direction, movement, and cyclical patterns of epidemics (Robertson et al., 2010; Delmelle et al., 2013a, 2013b). This approach could enhance understanding of transmission dynamics of STI caused by residential mobility of individuals with repeated cases. For this paper, we utilise the retrospective spacetime scan statistic (Kulldorff et al., 2005; Kulldorff, 2015), which is a widely used approach to identify space-time clusters of infectious disease (Coleman et al., 2009; Banu et al., 2014; Li et al., 2014; Mulatti et al., 2015; Desjardins et al., 2018). Furthermore, 3$\mathrm{D}$ visualisation approaches have been used to display space-time clusters of vector-borne diseases (Desjardins et al., 2018), crime (Nakaya and Yano, 2010; Cheng and Williams, 2012), and social media data (Cheng and Wicks, 2014). Although there are limitations with 3-D-visualisation such as crowding, occlusion, and static representation, it can improve the understanding of space-time clusters of disease and complements traditional 2D-approaches. The space-time scan statistics results and visualisations will enhance understanding of the impact of residential mobility in the prevalence and transmission dynamics of STI for purposes of protecting public health.

The purpose of this study is to develop a space-time scan statistic approach to examine the impact of residential mobility on the prevalence and transmission dynamics of STI. To achieve this, we examine a large surveillance data of reported chlamydia in Kalamazoo County, Michigan. In 2016, chlamydia constituted the highest number of reported STI with an incident rate of 497 per 100,000 in the U.S. (Centers for Disease Control and Prevention, CDC, 2017). We characterised residential mobility as the frequency of changes in the residential address reported for an individual in the surveillance data. We developed two scenarios, one with fixed residential address and one considering residential mobility and performed analysis using the space-time scan statistic. The resulting clusters and relative risk (RR) of infection were compared between scenarios to shed light on how residential mobility impacts the prevalence and transmission dynamics of sexually transmitted infections.

\section{Materials and Methods}

\section{Study population}

Data on all reported, confirmed cases of chlamydia from 2006 to 2014 in Kalamazoo County, Michigan (Figure 1) were retrieved from the Michigan Disease Surveillance System (MDSS). State law in Michigan requires all health care providers and laboratories to report chlamydia cases to MDSS for surveillance purposes. Each case in the MDSS contains an identification number, diagnosis date, patient name, age, gender, race, ethnic, residential address, and specific drug dosage for treatment information. Other useful information such as sexual orientation and partners were not available. The study was approved by the Western Michigan University Human Subjects Institutional Review Board (14-09-27).

Cases of chlamydia in Kalamazoo, Michigan, were used as the indicator STI for this analysis because health officials have identified chlamydia infection management as a critical issue.

The county ranks second among Michigan's 83 counties for chlamydia incidence, with approximately 2,000 cases per year. Nine years of reported chlamydia cases were included in the analysis to allow adequate examination of space-time trends that result from networks of individuals interacting through time in relation to a fairly stable background rate of disease.

Each chlamydia case in the MDSS was assigned a unique patient identifier. For patients with multiple reported chlamydia infections, a case was considered new if the second reported positive test occurred more than 14 days after the first positive test (Bernstein et al., 2004; Jennings et al., 2005; Owusu et al., 2018). We use the aforementioned threshold to identify secondary infections because chlamydia cases are symptomatic compared to other STIs (e.g. gonorrhoea) and the standard of care for the infection is a single-dose regimen with more than 95 percent efficacy; infection usually clears up after 14 days of treatment (Martin et al., 1992). Twenty records that corresponded to an individual having a second positive test within the 14-day window were therefore excluded. Furthermore, we excluded 9 cases of infants who tested positive for chlamydia infections at birth with the assumption that their infection was not through their own risky sexual activity. This enabled us to focus on the population at-risk of engaging in risky sexual activity. After these exclusions, the minimum age of infection in the dataset was 10 years and above. Finally, we attempted to geocoding all 14,982 records that fell within our study period. However, 204 cases were not geocoded because their addresses were at a post office box or missing other information such as house numbers.

\section{Measures}

Prior to geocoding, we examined the unique patient identifiers to identify patients with multiple cases of chlamydia infections (repeaters) during the study period. For repeaters, we determined if all cases were reported at the same residential address as the first reported case, or if multiple residential addresses were associated with an individual over time. We defined residential mobility as any change in the reported residential address in the surveillance data for individuals with repeated cases.

To determine the impact of residential mobility in the prevalence and transmission dynamics of chlamydia infections, we generated a new hypothetical dataset from the surveillance data. In this hypothetical data, we assumed stationary residential address for 
repeaters by assigning the same first reported residential address to all the cases they accumulated during the study period. For example, if the first residential address for a repeater was 200 Main St, and the cases accumulated was 4 , the same residential address of 200 Main St was assigned to all the remaining cases automatically. The temporal information corresponding to the date of diagnosis was not altered. In this way, we compared the analysis of data with and without residential mobility.

There was a total of 108 months from the start to the end of the study period (2006-2014). For each chlamydia case, we determined the month when the case was reported. The datasets in the two scenarios were geocoded separately in ArcGIS 10.5 (ESRI, Redlands, CA) a commercial geographic information system, to the 2014 street centreline obtained from the Kalamazoo County Planning Department. The geocoded cases of the two scenarios were placed on the 189 Kalamazoo County block groups of the 2010 U.S. Census. The block group centroid coordinates were then superimposed on the geocoded cases in the two datasets. This allowed aggregation at the block group level in a similar manner in studies focused on detecting STIs clusters (Jennings et al., 2005).

We obtained population data from the 2010 Census and American Community Survey (ACS) 5-year estimates for 2014 for the population 10 years and above considered at risk of chlamydia from the MDSS data for the county. The population 10 years and above data for 2010 was used for the reported cases from 2006 to 2010, while the ACS 2014 population for 10 years and above dataset was used for cases reported from 2011 to 2014. Using two population datasets will reduce the potential biases associated with assuming a fixed population for the block groups during the 9-year study period.

\section{Conceptualised scenarios: fixed residential address and residential mobility}

Figure 2 illustrates the concept of residential mobility with a purely hypothetical scenario. In Figure 2A, we use a three-dimensional visualisation, where the $\mathrm{X}$ and $\mathrm{Y}$ axes reflect the geographic space while the third axis represents the temporal dimension. We consider a situation when an individual with risky sexual behaviour (grey circle), becomes first infected with chlamydia. The arrows indicate the propagation of sexual activity while the red circle shows a newly infected individual. A green circle shows a repeat case of chlamydia. The figure shows two contrasting scenarios for two neighbourhoods.

In the first scenario, we assume all cases of chlamydia in respective of the number were diagnosed in the same neighbourhood of incidence. This is a hypothetical scenario of assuming fixed residential addresses. In the second scenario, the individual with repeat chlamydia cases (repeater) relocates into another neighbourhood without seeking treatment in the initial neighbourhood of incidence. While in the new neighbourhood, we hypothetically assume the individual engages in risky sexual behaviour and over time contributes to the formation of the sexual network. In our example, this second neighbourhood was without a case of chlamydia. However, because of residential mobility and continu-

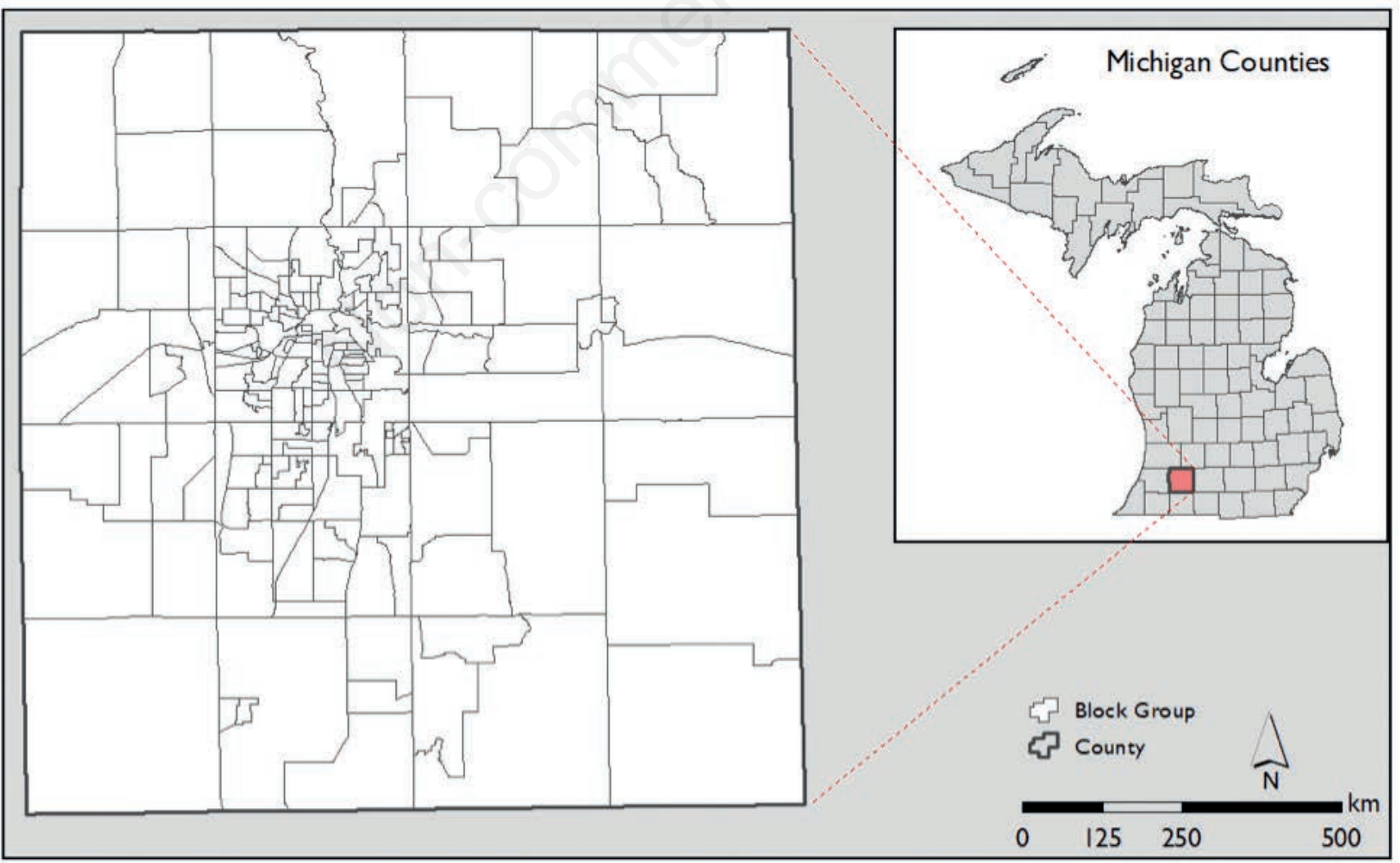

Figure 1. The study area Kalamazoo County, in the southwest of the state of Michigan. There are 189 block groups within the county. 
ous engagement of risky sexual behaviour, the dynamics of transmission of chlamydia is clearly seen to be changing over-time.

Figure $2 \mathrm{~B}$ and $\mathrm{C}$ summarises the number of cases observed per geographic unit, ignoring residential mobility (Figure 2B), and accounting for it (Figure 2C). In our example, a space-time cluster approach would detect the second neighbourhood as a cluster perhaps easily due to the high number of cases but would not account for the transmission dynamics. However, we hypothesise from this conceptualisation that, transmission dynamics of chlamydia at the neighbourhood level can be determined by finding the difference in the risk/rate of cases between assuming fixed residential and considering residential mobility.

\section{Space-time scan statistics}

To detect significant space-time clusters of chlamydia in Kalamazoo, we utilised the space-time scan statistic in SaTScan ${ }^{\mathrm{TM}}$ (Kulldorff et al., 2005). We ran the statistic to detect clusters for two scenarios, i) assuming no residential mobility during the study period, and ii) considering residential mobility of those who were infected with chlamydia. Conceptually, cylinders of varying spatial and temporal dimensions are moved throughout Kalamazoo County to scan for potential space-time clusters of chlamydia cases. Each cylinder is centred on the centroid of each block group, while the base of each cylinder represents the spatial dimension and the height denotes the temporal dimension. Conceptually, an infinite number of cylinders are produced of different spatial and temporal dimensions until an upper bound is reached. Each cylin- der is subsequently evaluated, which is later discussed. We set the maximum spatial and temporal size of the cylinders to $25 \%$ of the population at-risk and $50 \%$ of the study period, respectively. The maximum spatial and temporal scanning thresholds were selected to avoid the detection of very large clusters, while the threshold values correspond to what is typically chosen in the literature (Desjardins et al., 2018; Whiteman et al., 2019). Furthermore, each cylinder is a potential cluster, which contains observed and expected chlamydia cases. For this study, the number of expected cases is proportional to the total population corresponding to that census block group (i.e. total reported cases in Kalamazoo County divided by the population 10 years and above in each block group).

We use a Poisson-based probability model, where the number of chlamydia cases are assumed to be Poisson distributed according to the at-risk population in Kalamazoo. The null hypothesis $\left(\mathrm{H}_{0}\right)$ is that the model represents an inhomogeneous Poisson process with an intensity $\mu$ (proportional to the population 10 years and above considered at-risk of contracting chlamydia in the study area). The alternative hypothesis $\left(\mathrm{H}_{\mathrm{A}}\right)$ stipulates that the number of observed chlamydia cases exceeds the number of expected chlamydia cases. To address potential confounding by the population size, the statistic uses indirect standardisation (the expected number of cases in each block group under the null-hypothesis of the Poisson model). Each cylinder is evaluated using a maximum likelihood ratio test to evaluate $\mathrm{H}_{0}$ and $\mathrm{H}_{\mathrm{A}}$, which compares the observed and expected cases within a cylinder to the observed and expected cases outside of the cylinder and is defined in Eq. 1:

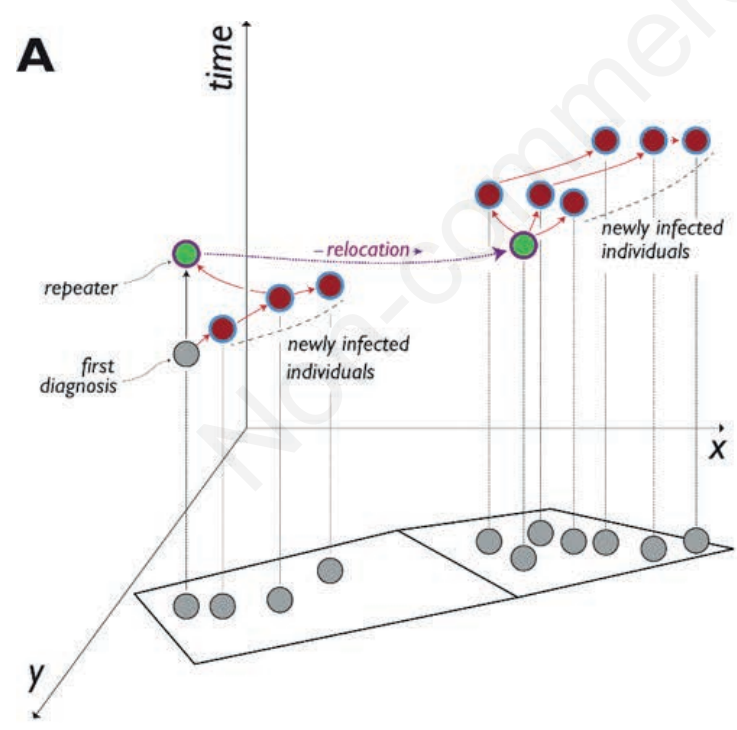

B number of cases per geographic unit without residential mobility

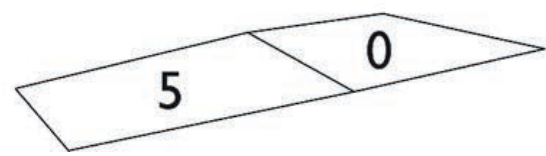

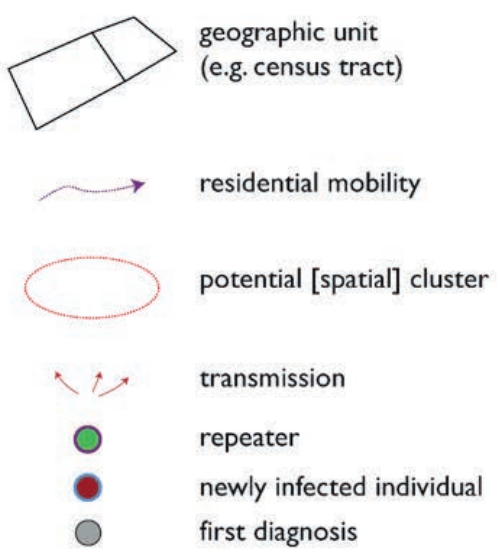

C number of cases per geographic unit with residential mobility (repeater moves)

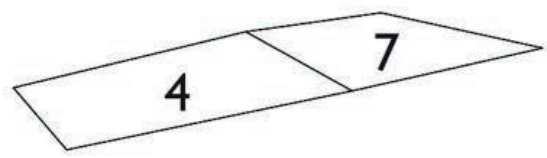

Figure 2. Illustration of residential mobility in a space-time context in (A) and its impact on the number of observed cases reported within various geographic units in $(B)$ and $(C)$. 


$$
\frac{L(Z)}{L_{0}}=\frac{\left(\frac{n_{Z}}{\mu(Z)}\right)^{n_{Z}}\left(\frac{N-n_{Z}}{N-\mu_{(Z)}}\right)^{N-n_{Z}}}{\left(\frac{N}{\mu(A)}\right)^{N}}
$$

where $n_{z}$ represents the number of observed cases in cylinder $Z$; $u(Z)$ the expected cases in cylinder $Z ; N$ the total number of observed cases in the study area across the entire study period; and $u(A)$ the total number of expected cases in the study area across the entire study period.

Cylinders with a likelihood ratio greater than 1 denote an elevated

risk of chlamydia cases, that is: $\frac{n_{Z}}{\mu(Z)}>\frac{N-n_{Z}}{N-\mu_{(Z)}}$

while the cylinder with the highest likelihood ratio is reported as the most likely cluster. The statistical significance of the clusters (P-value) is determined via 999 Monte Carlo simulations (comparing the observed cases to randomised expected cases); and we report clusters with a significance level $<0.05$. The resulting clusters have a minimum duration of one month and do not overlap spatially. Finally, we report and map the RR of chlamydia infections for both scenarios of no residential mobility and residential mobility for each block group belonging to a statistically significant space-time cluster, which is defined in Eq. 2:

$$
R R=\frac{o / e}{(O-o) /(O-e)}
$$

where $o$ is the number of observed chlamydia cases in a block group, $e$ the expected number of cases in a block group, and $O$ the total number of observed cases in our study area.

\section{Results}

Of the 15,012 chlamydia cases reported in Kalamazoo County from 1 January 2006 to 31 December 2014, 14,799 (98.4\%) satisfied the inclusion criteria for further analysis. The 14,799 chlamy- dia cases over the 9 years represented 10,228 unique individuals. Over the 9 years, 2.582 individuals reported repeat cases of chlamydia, representing 7,134 (48.3\%) cases, and 7,645 (51.7\%) were single cases of chlamydia.

Table 1 shows the demographic characteristics of geocoded repeaters and non-repeaters for chlamydia in the county. Few individuals reported 8 cases of chlamydia during the study period. Non-repeaters of chlamydia were more like to be females (62\%), non-Hispanic Whites (48\%) and were younger in the age group of 15-24 years $(68 \%)$. However, repeaters were more like to be females (71\%), non-Hispanic Blacks (64\%) and also in the younger age group of 15-24 years (81\%). In addition, high residential mobility was observed for females (76\%), non-Hispanic Blacks $(70 \%)$ in the younger age group of $15-24$ years $(80 \%)$.

Among the 2,582 repeaters of chlamydia, $41 \% \quad(n=1,063)$ reported only one residential address while $59 \%(n=1519)$ moved at least twice from their first reported residential address during the study period (Figure 3). In the case of individuals that moved at least twice from their first reported residential address, a total of $4,632(65 \%)$ of repeat cases can be attributed while $2502(35 \%)$ were repeat cases for those who did not move. This clearly indicates that the residential mobility of high-risk individuals

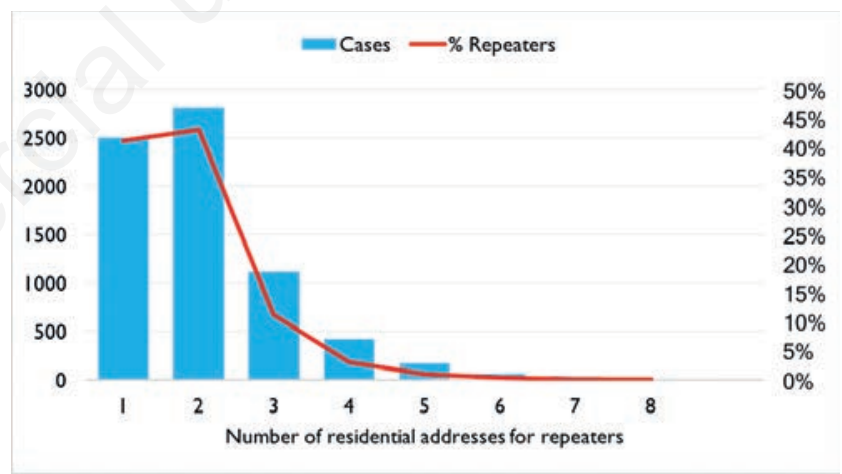

Figure 3. The number of cases and residential addresses for repeaters of chlamydia infections.

\begin{tabular}{|c|c|c|c|}
\hline Characteristic & $\begin{array}{l}\text { Non-repeaters } \\
\begin{array}{c}(\mathrm{N}=7,646) \\
\text { n }(\%)\end{array}\end{array}$ & $\begin{array}{c}\text { Repeaters } \\
(\mathrm{N}=2582) \\
\mathbf{n}(\%)\end{array}$ & $\begin{array}{l}\text { Individuals who moved at least once } \\
\qquad(\mathrm{N}=1519) \\
\mathrm{n}(\%)\end{array}$ \\
\hline \multicolumn{4}{|l|}{ Gender } \\
\hline Male & $2897(38)$ & $740(29)$ & $366(24)$ \\
\hline Female & $4748(62)$ & $1842(71)$ & $1153(76)$ \\
\hline \multicolumn{4}{|l|}{ Race/Ethnicity } \\
\hline Non-Hispanic white & $3678(48)$ & $760(29)$ & $373(25)$ \\
\hline Non-Hispanic black & $3105(41)$ & $1658(64)$ & $1059(70)$ \\
\hline Hispanic & $318(4)$ & $111(4)$ & $58(4)$ \\
\hline Other & $544(7)$ & $53(2)$ & $29(2)$ \\
\hline \multicolumn{4}{|l|}{ Age group } \\
\hline$\leq 14$ & $81(1)$ & $112(4)$ & $79(5)$ \\
\hline $15-24$ & $5212(68)$ & $2073(80)$ & $1237(81)$ \\
\hline $25-34$ & $1828(24)$ & $320(12)$ & $182(12)$ \\
\hline$\geq 35$ & $525(7)$ & & $21(1)$ \\
\hline
\end{tabular}

Table 1. Demographic characteristics of reported cases of chlamydia in Kalamazoo County, Michigan, 2006-2014. 
(repeaters) will have an impact on the prevalence and transmission dynamics of chlamydia in Kalamazoo County. Some individuals moved a maximum of 8 times during the study period (Figure 3 ).

\section{Significant space-time clusters}

We included all reported cases in the analysis to account for the space-time clusters considering residential mobility and for the hypothetical scenario with fixed residential addresses (without residential mobility). The results of the space-time scan statistics revealed three statistically significant $(\mathrm{P}<0.05)$ clusters for each scenario. These significant clusters varied by the number of block groups, estimated population 10 years and over at risk of chlamydia, start and end date, and RR (Table 2). Initially, cluster 3 in both scenarios comprised of a single block group. To protect the privacy of individuals in cluster 3 , we geomasked it by adding two extra block groups for reporting and visualisation purposes.

In general, the three significant space-time clusters found considering residential mobility were spatially similar to the scenario with fixed residential addresses (without residential mobility). In both instances, the largest and most likely cluster (cluster 1) located on the central east side of Kalamazoo County comprised of 40 block groups and encompassed of 37.4 square kilometres. The second largest cluster (cluster 2) was located on the west side of Kalamazoo County and comprised of 20 block groups and encompassed 36.2 square kilometres. The third cluster was located on the south side of Kalamazoo County and comprised of a single block group.

\section{3-D visualisation of significant space-time clusters}

The results of the space-time significant clusters are shown using a 3-D visualisation approach. Figure 4A shows the 3 spacetime clusters that were significant assuming with fixed residential addresses (without residential mobility). Cluster 1 began in February 2010 and lasted more than 4 years, until July 2014. Cluster 2 appeared nine months after Cluster 1 in November 2010 and lasted just over 4 years until December 2014. Cluster 3 started as early as June 2007 to October 2011. There was a temporal overlap among all thee clusters between February 2010 and October 2011 ( 1 year and 8 months); while clusters 1 and 2 overlapped temporally between November 2010 and July 2014 (3 years and 8 months). Figure 4B shows the 3 significant space-time clusters of chlamydia infections when residential mobility was considered. These clusters are spatially located in the same position as those clusters defined in the previous scenario. Temporally, however, cluster 3 began 3 years and one month later (July 2010) and lasted 3 years and 2 months longer (December 2014) when residential mobility was incorporated into the model. Furthermore, cluster 1 began one month later (March 2010) and lasted one month longer (August 2014); while cluster 2 began one month later (December 2010). The three clusters that accounted for residential mobility overlapped temporally between July 2010 and August 2014 (4 years and 1 month).

\section{The relative risk of significant space-time clusters}

Figure 5B shows the RR associated with the 3 significant space-time clusters of chlamydia with fixed residential addresses (without residential mobility). Out of the 40 block groups in cluster 1 , only 1 block group had a decreased risk of chlamydia infection $(\mathrm{RR}<1)$. The remaining 39 block groups in cluster 1 all had an increased risk of chlamydia infection $(R R>1)$. Out of the 23 block groups in cluster 2, 8 block groups had a decreased risk of chlamydia infection $(R R<1)$, while the remaining 15 block groups had an increased risk of chlamydia infections. Cluster $3 \mathrm{had}$ an increased risk of chlamydia infection $(\mathrm{RR}>1)$ during the entire study period.

Figure $5 \mathrm{C}$ shows the RR of the 3 significant space-time clusters with residential mobility. Out of the 40 block groups in cluster 1 , only 1 block group had a decreased risk of chlamydia infection $(\mathrm{RR}<1)$ while the remaining 39 block groups had an increased risk of chlamydia infection ( $R R>1)$. Out of the 23 block groups in cluster 2, 7 block groups had a decreased risk of chlamydia infection $(\mathrm{RR}<1)$ while the remaining 16 block groups had an increased risk of chlamydia infection. Cluster 3 had an increased risk of chlamydia infection $(R R>1)$ during the entire study period.

\section{Impact of residential mobility}

We computed the change in RR quantified from the difference between the significant space-time clusters considering the residential mobility and the hypothetical scenario with fixed residential addresses (without residential mobility). This quantified value represents whether there is a positive, negative or no impact of residential mobility in the clusters detected.

Figure 6 depicts the change in relative risk (CRR) that was

Table 2. Results of SaTScan space-time analysis without residential mobility and with residential mobility for all reported chlamydia cases in Kalamazoo County, 2006-2014.

\begin{tabular}{|c|c|c|c|c|c|c|}
\hline \multirow[b]{2}{*}{ Cluster } & \multicolumn{3}{|c|}{ Without residential mobility } & \multicolumn{3}{|c|}{ With residential mobility } \\
\hline & 1 & 2 & $3^{*}$ & 1 & 2 & $3^{*}$ \\
\hline Block groups & 40 & 23 & $3^{*}$ & 40 & 23 & $3^{*}$ \\
\hline Estimated Population & 39,094 & 39,849 & $3.197^{*}$ & 39,094 & 39,849 & $3,197^{*}$ \\
\hline Start date & 02-2010 & $11-2010$ & 06-2007 & 03-2010 & $12-2010$ & $07-2010$ \\
\hline End date & 07-2014 & $12-2014$ & 10-2011 & 08-2014 & $12-2014$ & $12-2014$ \\
\hline Observed cases & 3498 & 1718 & 118 & 3371 & 1764 & 149 \\
\hline Expected cases & 1127 & 1093 & 26 & 1126 & 1072 & 27 \\
\hline Relative risk & 3.76 & 1.65 & 4.6 & 3.58 & 1.73 & 5.5 \\
\hline LLR & 1810.4 & 166.2 & 87.5 & 1647.5 & 204.3 & 131.5 \\
\hline P-value & $<.01$ & $<.01$ & $<.01$ & $<.01$ & $<.01$ & $<.01$ \\
\hline
\end{tabular}

LLR, Log Likelihood Ratio. *For reporting purposes, geomasked by adding 2 more block groups to a single block group to prevent disclosure. 
used to quantify the impact of residential mobility. Of the 40 block groups in cluster 1,63 percent of the block groups $(n=25)$ had a decrease $(\mathrm{CRR}<0)$ in chlamydia risk after high-risk individuals moved-out from that cluster. Furthermore, 18 percent of the block groups $(n=8)$ in cluster 1 had no change in chlamydia risk $(C R R=0)$. The remaining 19 percent of block groups $(n=8)$ in cluster 1 had an increased change in risk $(C R R>0)$ after high-risk individuals moved-into the cluster. Out of the 23 block groups in cluster 2,22 percent of the block groups $(n=5)$ had a decrease in $(\mathrm{CRR}<0)$ chlamydia risk after high-risk individuals moved-out from the cluster. Approximately 26 percent of the block groups $(n=6)$ in cluster 2 had no change in its chlamydia risk $(C R R=0)$. The remaining 52 percent of block groups $(n=12)$ in cluster 2 had an increase in risk $(C R R>0)$ after high-risk individuals moved-into the cluster. Cluster 3 had an increase in risk $(C R R>0)$ through the entire study period after high-risk individuals moved-into the cluster. Table 3 summarises the origin and destination (O-D) movements between and within the clusters for individuals with repeaters of chlamydia. Considering within the cluster, individuals were more likely to move into cluster $1(n=1601)$, followed by cluster $2(n=349)$, with least number of moves in cluster $3(n=41)$. Between the clusters, individuals were more likely to move from cluster 2 into cluster $1(n=121)$ compared to moving from cluster 1 into cluster $2(n=91)$. Moving into cluster $3(n=15)$ were mostly from individuals whose origin were in cluster 1.
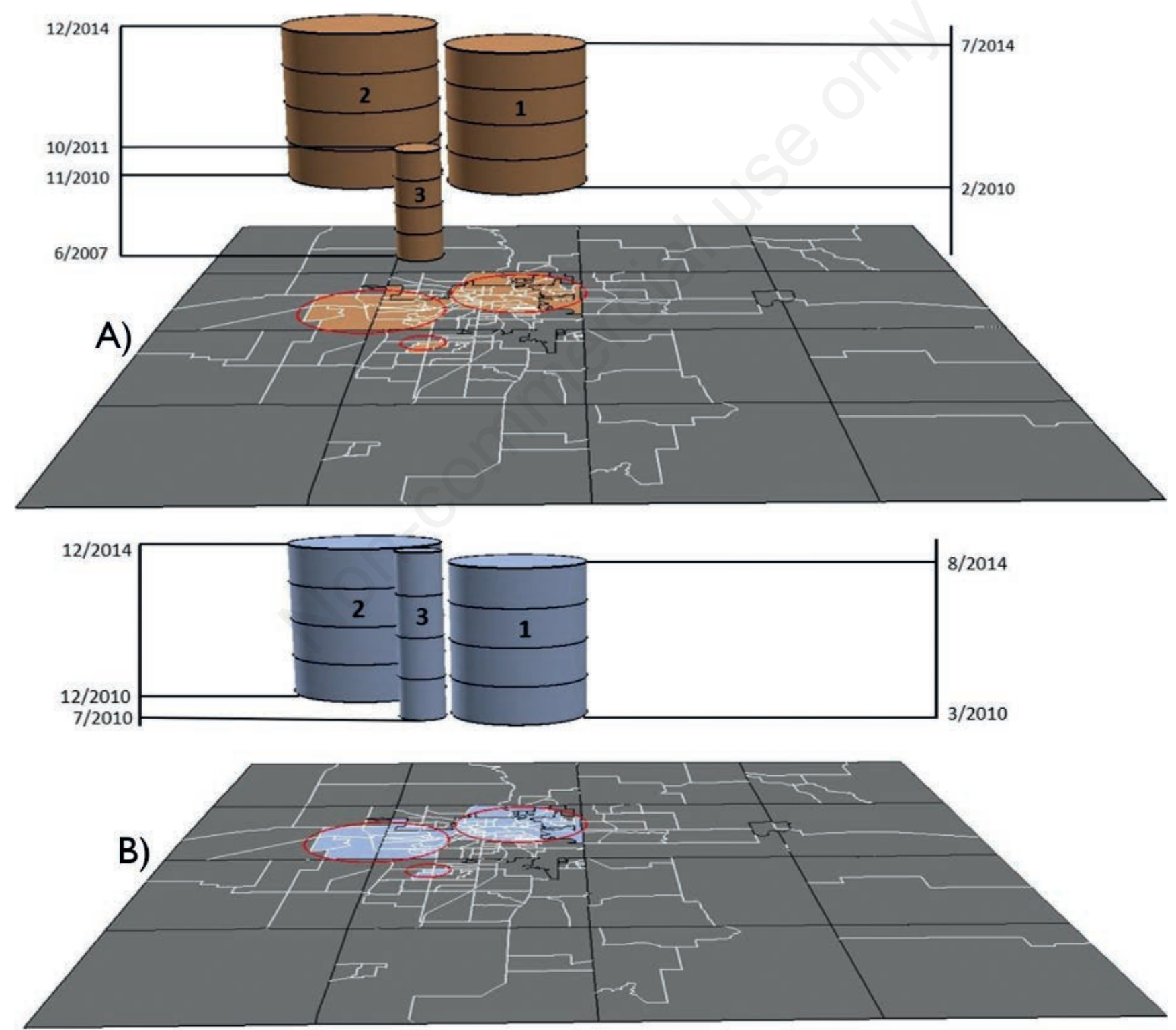

Figure 4. 3-D visualisation of statistically significant space-time clusters of chlamydia infections for without residential mobility (A) and with residential mobility (B) from 2006 to 2014. 


\section{Discussion}

The results indicate that there are three main space-time clusters of chlamydia in Kalamazoo County. Within the clusters, living in areas with a high influx of repeaters of chlamydia increased the risk of infection for all individuals. Considering that we initially observed relatively low RR in cluster 2 and 3 under the assumption of no residential mobility, it appears that the influx of repeaters may have induced risky sexual behaviours in these areas. This is particularly troubling as it appears that the transmission dynamics of chlamydia are diffusing from the most likely core area (cluster 1). This finding is consistent with other results that found that high mobility of high-risk individuals leads to increased STIs in historically low-risk areas (Aral, 2000; Lichtenstein et al., 2008; Apostolopoulos et al., 2015; Cassels et al., 2017).

The spatially similar significant space-time clusters under the two scenarios of with residential mobility and without residential mobility suggests that individuals with chlamydia infections live or move into specific neighbourhoods already vulnerable to STIs. In addition, the finding may also suggest a formation of a localised structured network of sex partners within specific neighbourhoods. If so, this is consistent with other studies that found that individu-

Table 3. Summary of origin and destination movements between and within the clusters.

\begin{tabular}{lccc} 
Origin & \multicolumn{3}{c}{ Destination } \\
1 & 1 & 2 & 3 \\
2 & 1601 & 91 & 15 \\
\hline 3 & 121 & 349 & 4 \\
\hline
\end{tabular}

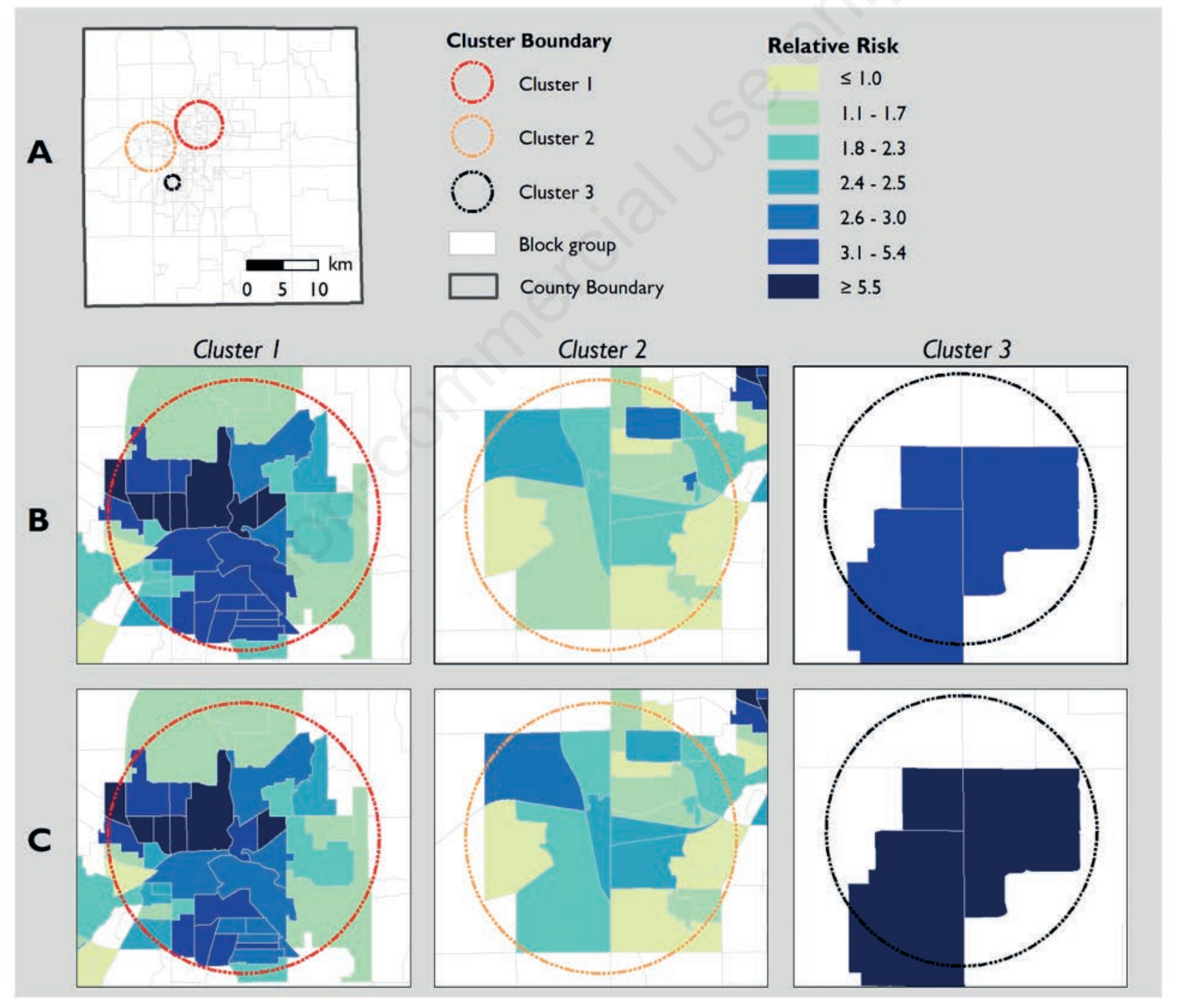

Figure 5. The relative risk of significant space-time clusters. Spatial location of all the 3 clusters (A), without residential mobility (B), and with residential mobility (C) from 2006 to 2014. 
als with STIs stay near their sexual partners (Zenilman et al., 1999; Gunn et al., 2000; Bernstein et al., 2004). However, we found differences in temporal information when we compare the scenarios with residential mobility and with fixed residency. We recommend STI studies to take into consideration both the spatial and temporal dynamics in order to implement cost-effective, targeted interventions. Furthermore, we found that repeaters of chlamydia were more likely to be non-Hispanic blacks, females and mostly in the age group of 15-24 years. This finding is consistent to the breadth of literature on characteristics of the population with a high risk of acquiring STIs in the U.S. (Bernstein et al., 2004; Jennings et al., 2005; Centers for Disease Control and Prevention, CDC, 2017). However, we also found these populations are most likely to move neighbourhoods with high number rates of STI. This increases their risk of getting STI particularly if they engage in risky sexual behaviours in the new neighbourhood. Owusu et al. (2018) modelled the vulnerability of STIs in Kalamazoo County and found that race and socioeconomic status were the most important contextual variables to consider for targeted interventions. It appears most transmission and prevalence of STI were moderated by residential mobility because of economic hardships in those neighbourhoods. Structural policies that can lead to an improvement in housing tenure could be important to reduce the transmission of STI in low socioeconomic neighbourhoods.

A strategy to reduce the burden of chlamydia in the community is to tailor interventions to neighbourhoods experiencing a high influx of repeaters of chlamydia. Such an approach can help track the transmission dynamics of the STI and will guide strategic interventions to those most vulnerable to risky sexual behaviour. This will further enable stakeholders to understand areas in the community in need of heightened STI resources. Although this current county-level study may not be generalisable to all settings, the large sample size and the findings are noteworthy because it suggests that prevalence and transmission dynamics is associated with changing residential patterns of repeaters of chlamydia.

Given that other unknown underlying factors can prompt an individual to move in or out of a neighbourhood, it is important that future studies examine why repeaters of chlamydia are consistently moving over time. The paucity of research on the impact of residential mobility on health outcomes can be explained by the difficulty to secure hospital discharge information from which it is possible to determine a patient's movement over time. Ignoring the impact of residential mobility in health studies may lead to inaccurate estimation of the number of cases in each geographic unit, affecting spatial and space-time statistical results - such as cluster detection - ultimately leading to wrong identification of high-risk regions (Kirby et al., 2017). We anticipate an approach like this study may inspire further research in this area. Our study had several limitations. First, our dataset does not include the exact time corresponding to when the residential move occurred. Second, we conducted our analysis at the block group level, and more accurate results could be obtained at the block or at the individual level. This issue is known in health geography as the modifiable area unit problem (Openshaw and Taylor, 1979). On the other hand, conducting our analysis at the block group level guarantees a certain level of privacy but limited our ability to find spatial variation in residential mobility. Despite these limitations, our study exhibited several strengths. First, our study was able to conceptualise and quantify the risk associated with residential mobility using spacetime scan statistical approach to determine the prevalence and transmission dynamics of STIs at the county level. Second, our study took advantage of the large sample size from surveillance data collected for nine years to demonstrate the importance of accounting for residential mobility to explain the transmission of STI to new areas. Finally, we supplemented traditional 2D visualisation approaches with 3-D visualisations to enable stakeholders in the county to understand the epidemic to better plan STI interventions with their limited resources.

\section{Change in Relative Risk}

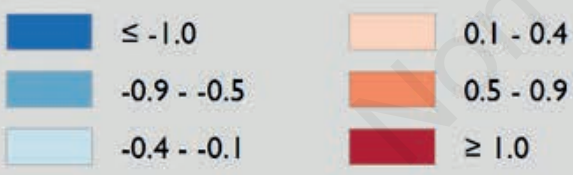

Cluster I
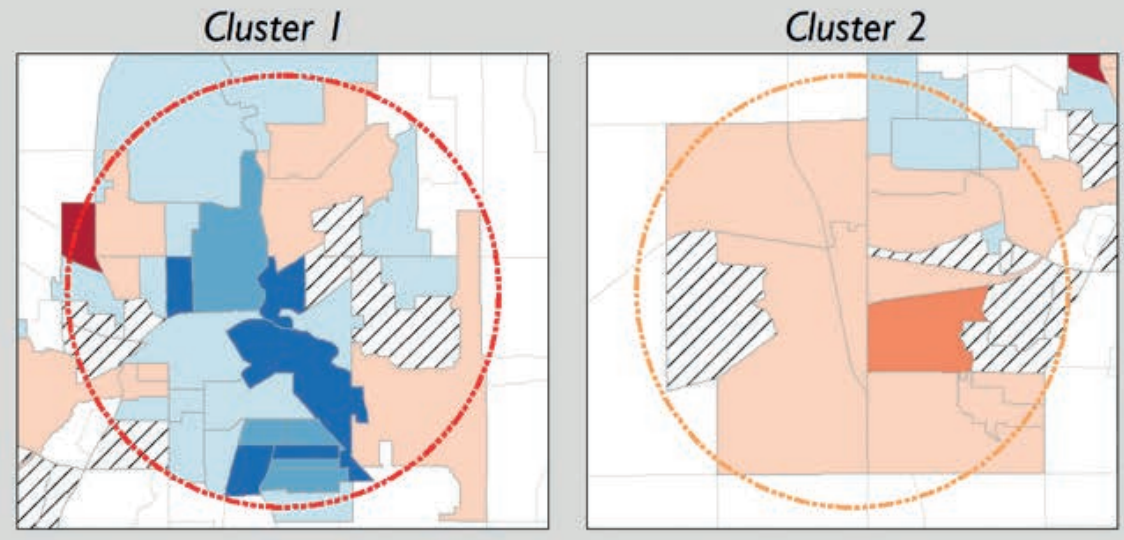

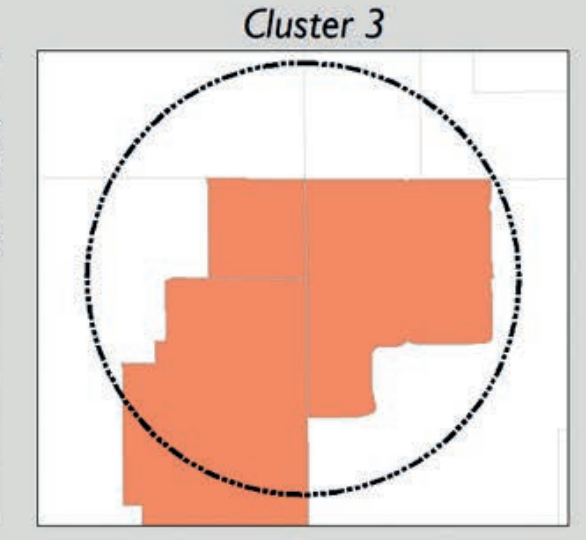

Figure 6. Change in relative risk of significant space-time clusters considering residential mobility and without residential mobility. 


\section{Conclusions}

Given the high incidence of chlamydia in the United States in general (and Kalamazoo County, Michigan, in particular), accounting for residential mobility will enhance understanding of transmission dynamics of STI. Furthermore, it also has the advantage to make the space-time clusters more realistic and accurate. The findings indicate STI clusters last longer in places with a high number of residential mobility because individuals in need of interventions are likely to move before full implementation of public health interventions. We recommend that strategic interventions such as setting up screening sites and creating public awareness take into consideration residential mobility in order to reach the targeted population in a timely manner. This can be facilitated by examining residential patterns of individuals with high-risk of STI in surveillance data. This should be done with care to avoid disclosure of privacy particularly in the presentation of the results.

\section{References}

Apostolopoulos Y, Sönmez S, Lemke MK, Rothenberg RB, 2015. Mapping US long-haul truck drivers' multiplex networks and risk topography in inner-city neighborhoods. Health Place 34:9-18

Aral SO, 2000. Behavioral aspects of sexually transmitted diseases: core groups and bridge populations. Sex Transm Dis 27:327-8.

Banu S, Hu W, Guo Y, Naish S, Tong S, 2014. Dynamic spatiotemporal trends of dengue transmission in the Asia-Pacific region, 1955-2004. PloS One 9:e89440.

Bernstein KT, Curriero FC, Jennings JM, Olthoff G, Erbelding EJ, Zenilman J, 2004. Defining core gonorrhea transmission utilizing spatial data. Am J Epidemiol 160:51-8.

Bush KR, Henderson EA, Dunn J, Read RR, Singh A, 2008. Mapping the core: chlamydia and gonorrhea infections in Calgary, Alberta. Sex Transm Dis 35:291-7.

Cassels S, Jenness SM, Biney AAE, Dodoo FN, 2017. Geographic mobility and potential bridging for sexually transmitted infections in Agbogbloshie, Ghana. Soc Sci Med 184:27-39.

Centers for Disease Control and Prevention, CDC, 2017. Sexually transmitted disease surveillance 2016. U.S. Department of Health and Human Services, Atlanta, USA.

Cheng T, Wicks T, 2014. Event detection using Twitter: a spatiotemporal approach. PloS One 9:e97807.

Cheng T, Williams D, 2012. Space-time analysis of crime patterns in central London. Int Arch Photogramm Remote Sens Spat Inf Sci 39:47-52.

Coleman M, Coleman M, Mabuza AM, Kok G, Coetzee M, Durrheim DN, 2009. Using the SaTScan method to detect local malaria clusters for guiding malaria control programmes. Malar J 8:68.

Delmelle E, Casas I, Rojas JH, Varela A, 2013a. Spatio-temporal patterns of dengue fever in Cali, Colombia. Int J Appl Geospat Res 4:58-75.

Delmelle E, Kim C, Xiao N, Chen W, 2013b. Methods for spacetime analysis and modeling: an overview. Int J Appl Geospat Res 4:1-18.

Desjardins M, Whiteman A, Casas I, Delmelle E, 2018. Space-time clusters and co-occurrence of chikungunya and dengue fever in
Colombia from 2015 to 2016. Acta Trop 185:77-85.

Fergusson DM, Horwood LJ, 1997. Early onset cannabis use and psychosocial adjustment in young adults. Addiction 92:27996.

Gesink DC, Sullivan AB, Miller WC, Bernstein KT, 2011. Sexually transmitted disease core theory: roles of person, place, and time. Am J Epidemiol 174:81-9.

Gunn RA, Fitzgerald S, Aral SO, 2000. Sexually transmitted disease clinic clients at risk for subsequent gonorrhea and chlamydia infections: possible 'core' transmitters. Sex Transm Dis 27:343-9.

Jacquez GM, Barlow J, Rommel R, Kaufmann A, Rienti M, AvRuskin G, Rasul J, 2013. Residential mobility and breast cancer in Marin County, California, USA. Int J Environ Res Public Health 11:271-95.

Jacquez GM, Kaufmann A, Meliker J, Goovaerts P, AvRuskin G, Nriagu J, 2005. Global, local and focused geographic clustering for case-control data with residential histories. Environ Health 4:4.

Jacquez GM, Meliker J, Kaufmann A, 2007. In search of induction and latency periods: Space-time interaction accounting for residential mobility, risk factors and covariates. Int $\mathbf{J}$ Health Geogr 6:35.

Jelleyman T, Spencer N, 2008. Residential mobility in childhood and health outcomes: a systematic review. J Epidemiol Commun Health 62:584-92.

Jennings JM, Curriero FC, Celentano D, Ellen JM, 2005. Geographic identification of high gonorrhea transmission areas in Baltimore, Maryland. Am J Epidemiol 161:73-80.

Kulldorff M, 2015. SaTScan User Guide v9. 4. National Cancer Institute, New York, USA.

Kirby RS, Delmelle E, Eberth JM, 2017. Advances in spatial epidemiology and geographic information systems. Ann Epidemiol 27:1-9.

Kulldorff M, Heffernan R, Hartman J, Assunçao E, Mostashari F, 2005. A space-time permutation scan statistic for disease outbreak detection. PLoS Med 2:e59.

Li J, Kolivras KN, Hong Y, Duan Y, Seukep SE, Prisley SP, Campbell JB, Gaines DN, 2014. Spatial and temporal emergence pattern of Lyme disease in Virginia. Am J Trop Med Hyg 91:1166-72.

Lichtenstein B, Hook EW 3rd, Grimley DM, St. Lawrence JS, Bachmann LH, 2008. HIV risk among long-haul truckers in the USA. Cult Health Sex 10:43-56.

Martin DH, Mroczkowski TF, Dalu ZA, McCarty J, Jones RB, Hopkins SJ, Johnson RB, 1992. A controlled trial of a single dose of azithromycin for the treatment of chlamydial urethritis and cervicitis. The Azithromycin for Chlamydial Infections Study Group. N Engl J Med 327:921-5.

Morris T, Manley D, Northstone K, Sabel CE, 2016. On the move: exploring the impact of residential mobility on cannabis use. Soc Sci Med 168:239-48.

Mulatti P, Mazzucato M, Montarsi F, Ciocchetta S, Capelli G, Bonfanti L, Marangon S, 2015. Retrospective space-time analysis methods to support West Nile virus surveillance activities. Epidemiol Infect 143:202-13.

Nakaya T, Yano K, 2010. Visualising crime clusters in a space-time cube: an exploratory data-analysis approach using space-time Kernel density estimation and scan statistics. Transact GIS 14:223-39.

Neaigus A, Jenness SM, Reilly KH, Youm Y, Hagan H, Wendel T, 
Gelpi-Acosta C, 2016. Community sexual bridging among heterosexuals at high-risk of HIV in New York city. AIDS Behav 20:722-36.

Nguyen NT, Nguyen HT, Trinh HQ, Mills SJ, Detels R, 2009. Clients of female sex workers as a bridging population in Vietnam. AIDS Behav 13:881-91.

Openshaw S, Taylor PJ, 1979. A million or so correlation coefficients, three experiments on the modifiable areal unit problem. In: Wrigley N, ed. Statistical applications in the spatial sciences. Pion, London, UK, pp 127-144.

Owusu C, Baker KM, Paul R, Curtis AB, 2018. Modelling individual vulnerability to sexually transmitted infections to optimise intervention strategies: analysis of surveillance data from Kalamazoo County, Michigan, USA. Sex Transm Infect 94:353-8.

Owusu-Edusei K Jr, Chesson HW, Gift TL, Tao G, Mahajan R, Ocfemia MC, Kent CK, 2013. The estimated direct medical cost of selected sexually transmitted infections in the United States, 2008. Sex Transm Dis 40:197-201.

Robertson C, Nelson TA, MacNab YC, Lawson AB, 2010. Review of methods for space-time disease surveillance. Spat Spatiotemporal Epidemiol 1:105-16.

Satta A, Stivala A, Garozzo A, Morello A, Perdichizzi A, Vicari A, Salmeri M, Calogero AE, 2005. Experimental Chlamydia trachomatis infection causes apoptosis in human sperm. Hum Reprod 21:134-7.
Schafft KA, 2006. Poverty, residential mobility, and student transiency within a rural New York school district. Rural Sociol 71:212-31.

Stack S, 1994. The effect of geographic mobility on premarital sex. J Marriage Fam 56:204-8.

Stratford D, Ellerbrock TV, Akins JK, Hall HL, 2000. Highway cowboys, old hands, and Christian truckers: risk behavior for human immunodeficiency virus infection among long-haul truckers in Florida. Soc Sci Med 50:737-49.

Thomas JC, Torrone EA, Browning CR, 2010. Neighborhood factors affecting rates of sexually transmitted diseases in Chicago. J Urban Health 87:102-12.

van Aar F, den Daas C, van der Sande M, Soetens L, de Vries H, van Benthem B, 2016. Outbreaks of syphilis among men who have sex with men attending STI clinics between 2007 and 2015 in the Netherlands: a space-time clustering study. Sex Transm Infect 93:390-5.

Whiteman A, Desjardins MR, Eskildsen GA, Loaiza JR, 2019. Integrating vector surveillance data to improve space-time risk estimation of dengue fever in Panama. PLoS Negl Trop Dis 13:e0007266.

Zenilman JM, Ellish N, Fresia A, Glass G, 1999. The geography of sexual partnerships in Baltimore: applications of core theory dynamics using a geographic information system. Sex Transm Dis 26:75-81. 